\title{
Gravitational wave detection on the Moon and the moons of Mars
}

\author{
Ho Jung Paik and Krishna Yethadka Venkateswara \\ Department of Physics, University of Maryland, College Park, MD 20742, USA
}

\begin{abstract}
The Moon and the moons of Mars should be extremely quiet seismically and could therefore become sensitive gravitational wave detectors, if instrumented properly. Highly sensitive displacement sensors could be deployed on these planetary bodies to monitor the motion induced by gravitational waves. A superconducting displacement sensor with a 10-kg test mass cooled to $2 \mathrm{~K}$ will have an intrinsic instrument noise of $10^{-16} \mathrm{~m} \mathrm{~Hz}^{-1 / 2}$. These sensors could be tuned to the lowest two quadrupole modes of the body or operated as a wideband detector below its fundamental mode. An interesting frequency range is $0.1 \sim 1 \mathrm{~Hz}$, which will be missed by both the ground detectors on the Earth and LISA and would be the best window for searching for stochastic background gravitational waves. Phobos and Deimos have their lowest quadrupole modes at $0.2 \sim 0.3 \mathrm{~Hz}$ and could offer a sensitivity $h_{\min } \leq 10^{-22} \mathrm{~Hz}^{-1 / 2}$ within their resonance peaks, which is within two orders of magnitude from the goal of the Big Bang Observer (BBO). The lunar and Martian moon detectors would detect many interesting foreground sources in a new frequency window and could serve as a valuable precursor for BBO.

PACS numbers: $04.80 . \mathrm{Nn}, 95.55 . \mathrm{Ym}$
\end{abstract}

\section{Introduction}

Due to lack of plate tectonics and its spin locked to its revolution, the Moon is very quiet seismically. Its total seismic energy release per year is estimated to be $10^{9}$ times lower than the Earth (Goins et al 1981). Moonquakes are driven mainly by tidal deformation due to the orbit eccentricity (0.05) and occur within a few days from the perigee (Lammlein et al 1974). With the absence of ocean waves and winds, the seismic noise level between moonquakes may be extremely low. Conditions should be similar on the moons of Mars. Of the two Martian moons, Deimos is especially interesting since it is farther out from Mars and its orbit eccentricity is only 0.003 . This leads to an interesting possibility that the entire Moon and the moons of Mars could be instrumented as sensitive resonant-mass gravitational wave (GW) antennas.

This is exactly what Weber attempted to do in his ill-fated Apollo 17 Lunar Surface Gravimeter Experiment. His gravimeter was designed to monitor free oscillations of the Moon, possibly induced by GWs from astrophysical sources. In view of NASA's new initiative of Moon/Mars exploration, it is timely to revisit the idea of using planetary bodies as GW detectors.

\section{Spherical antenna with superconducting displacement sensors}

Although Phobos and Deimos are highly irregular in shape, the Moon is nearly spherical. A spherical antenna has the advantage of having a uniform cross section for all sky. Further, the amplitudes of its five degenerate quadrupole modes can be combined to determine the four unknowns: source direction $(\theta, \phi)$ and wave polarization $\left(h_{+}, h_{-}\right)$, and the remaining degree of freedom can be used to discriminate against non-GW disturbances (Wagoner and Paik 1977). Only quadrupole modes couple to spin-2 GWs. The TIGA (truncated icosahedral gravitational-wave antenna) configuration of six radial transducers (see Figure 1) has been shown to preserve the 


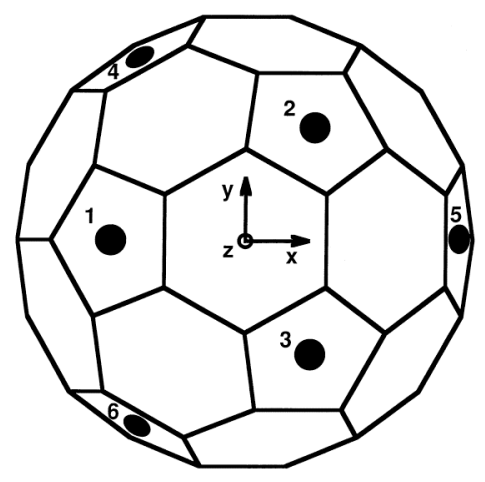

Figure 1. TIGA configuration.

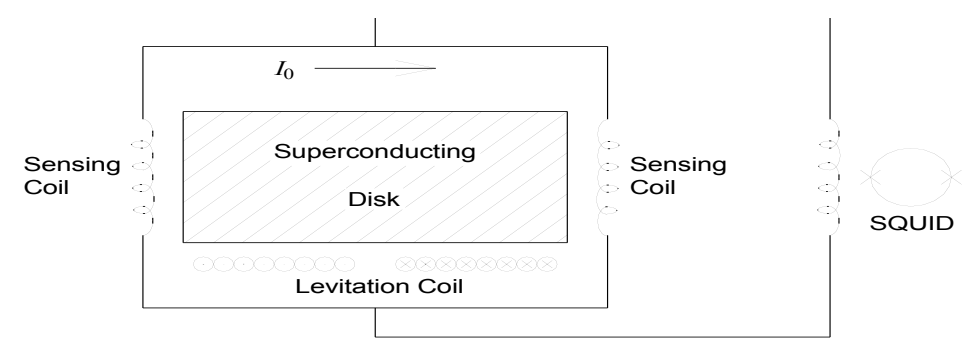

Figure 2. Schematic of superconducting displacement sensor.

five-fold degeneracy and omni-directionality (Johnson and Merkowitz 1993). We find that the same holds true for tangential transducers.

A highly sensitive tangential, or horizontal, displacement sensor could be constructed by combining a magnetically levitated test mass with a superconducting inductive transducer (Paik 1976). Figure 2 shows a schematic of the superconducting displacement sensor. The horizontal displacement of the levitated superconducting disk modulates the magnetic flux produced in each sensing coil, inducing a flux in the SQUID input coil proportional to the displacement.

The power spectral density of its intrinsic instrument noise can be shown to be

$$
S_{x}(f)=\frac{4}{m \omega^{4}}\left\{k_{B} T \frac{\omega_{0}}{Q_{0}}+E_{A}(f) \frac{1}{2 \beta \eta \omega_{0}^{2}}\left[\left(\omega_{0}^{2}-\omega^{2}\right)^{2}+\left(\frac{\omega_{0} \omega}{Q_{0}}\right)^{2}\right]\right\},
$$

where $m, f_{0}=\omega_{0} / 2 \pi$, and $Q_{0}$ are the mass, resonance frequency, and quality factor of the test mass; $\beta, \eta$, and $E_{A}(f)$ are the transducer energy coupling constant, amplifier coupling efficiency, and SQUID energy resolution; and $f=\omega / 2 \pi$ is the signal frequency, respectively. With somewhat optimistic but feasible values of $m=10 \mathrm{~kg}, f_{0}=0.3 \mathrm{~Hz}, T=2 \mathrm{~K}, Q_{0}=10^{8}, 2 \eta \beta=0.5, E_{A}(f)$ $=10^{-31} \mathrm{~J} \mathrm{~Hz}^{-1}$, we find $S_{x}^{1 / 2}(f) \approx 10^{-16} \mathrm{~m} \mathrm{~Hz}^{-1 / 2}$ at $f=0.3 \mathrm{~Hz}$. This displacement sensor would be $10^{6}$ times more sensitive than the Apollo lunar seismometers (Lammlein et al 1974).

\section{Two modes of detector operation}

There are two ways of operating a planetary gravitational wave detector: (1) as a wideband detector below its lowest mode, where the planetary body acts as a rigid platform, and (2) as a resonant detector, by tuning the displacement sensors to the fundamental $(n=1)$ or second harmonic $(n=2)$ quadrupole mode $(\ell=2)$, which couple strongly with GWs (Lobo 1995).

To obtain approximate sensitivities of the spherical detector in the two modes of operation, the detector response is approximated as that of a cylinder and the GW power spectral density is summed over the six displacement sensors with appropriate geometric factors. The detector response can be written as

$$
(\ddot{x}-\ddot{\xi})+\tau_{0}^{-1}(\dot{x}-\dot{\xi})+\omega_{0}^{2}(x-\xi)=\frac{1}{2} \ddot{h} R-\ddot{\xi},
$$

where $x$ and $\xi$ are the displacement of the test mass and the planet's surface, respectively, and $R$ is the radius of the planet. 
Below its fundamental frequency $\left(f<f_{1}\right)$, a planetary body will act as a rigid platform, which does not respond to GWs or the moonquakes: $\xi=\dot{\xi}=\ddot{\xi}=0$. To maximize the sensitivity of the wideband detector, the test masses must be almost free $\left(f_{0}<f\right)$. The GW power spectral density summed over six sensors then becomes

$$
S_{h}(f) \approx 10\left(\frac{2}{R}\right)^{2} S_{x}(f) .
$$

It is interesting to compare the potential sensitivity of a wideband spherical detector with that of LISA. The Moon has $f_{1} \approx 10^{-3} \mathrm{~Hz}$. If the displacement sensors are tuned to $f_{0}=10^{-4} \mathrm{~Hz}$, the sensor-noise-limited detector sensitivity becomes $S_{h}^{1 / 2}(f) \approx\left(10^{-4} \mathrm{~Hz} / f\right)^{2} 3 \times 10^{-18} \mathrm{~Hz}^{-1 / 2}$ for $f=10^{-4}$ $\sim 10^{-3} \mathrm{~Hz}$. This is comparable to the sensitivity of LISA. Deimos, which is 270 times smaller in diameter than the Moon, will permit a wideband operation below $f_{1} \approx 0.3 \mathrm{~Hz}$. The sensitivity is again found to be comparable with LISA in the frequency band $f=0.1 \sim 0.3 \mathrm{~Hz}$.

Above the fundamental frequency $\left(f>f_{1}\right)$, the driving terms in Eq. (2) cancel except at the quadrupole mode frequencies $\omega_{n}$ (Chen and Thorne 2004), where we obtain

$$
x-\xi=-0.2 i \omega_{1}^{2} h R \frac{\omega^{2}}{\omega_{n}^{2}-\omega^{2}+i \omega \omega_{n} / Q_{n}} \frac{\omega^{2}}{\omega_{0}^{2}-\omega^{2}+i \omega \omega_{0} / Q_{0}} .
$$

The GW power spectral density summed over six sensors becomes

$$
S_{h}(f) \approx \frac{10}{\left(0.2 \omega_{1}^{2} R\right)^{2}}\left[\left(\omega_{n}^{2}-\omega^{2}\right)^{2}+\left(\frac{\omega \omega_{n}}{Q_{n}}\right)^{2}\right] S_{x}(f) .
$$

With $Q_{1} \approx 2000$, as measured on the Moon, this leads to the sensor-noise-limited sensitivities for the Moon and Deimos: $S_{h}^{1 / 2}(f) \approx 2 \times 10^{-21} \mathrm{~Hz}^{-1 / 2}$ at $f=f_{1}=10^{-3} \mathrm{~Hz}$ and $S_{h}^{1 / 2}(f) \approx 3 \times 10^{-20} \mathrm{~Hz}^{-1 / 2}$ at $f=f_{1}=0.3 \mathrm{~Hz}$, respectively.

Figure 3 compares the sensitivities of the Moon and Deimos with those of LIGO, LISA, and BBO (Big Bang Observer). Also shown in the figure are expected signals from foreground blackhole, neutron star, and white dwarf binaries, as well as the stochastic background from the Big Bang. Deimos looks especially attractive because it will open a new frequency window between the ground detectors and LISA, and its sensitivity comes within two orders of magnitude from the goal of $\mathrm{BBO}$, albeit within a very narrow bandwidth (Phinney 2004). Such a detector will detect many interesting foreground sources and will serve as a valuable precursor to $\mathrm{BBO}$.

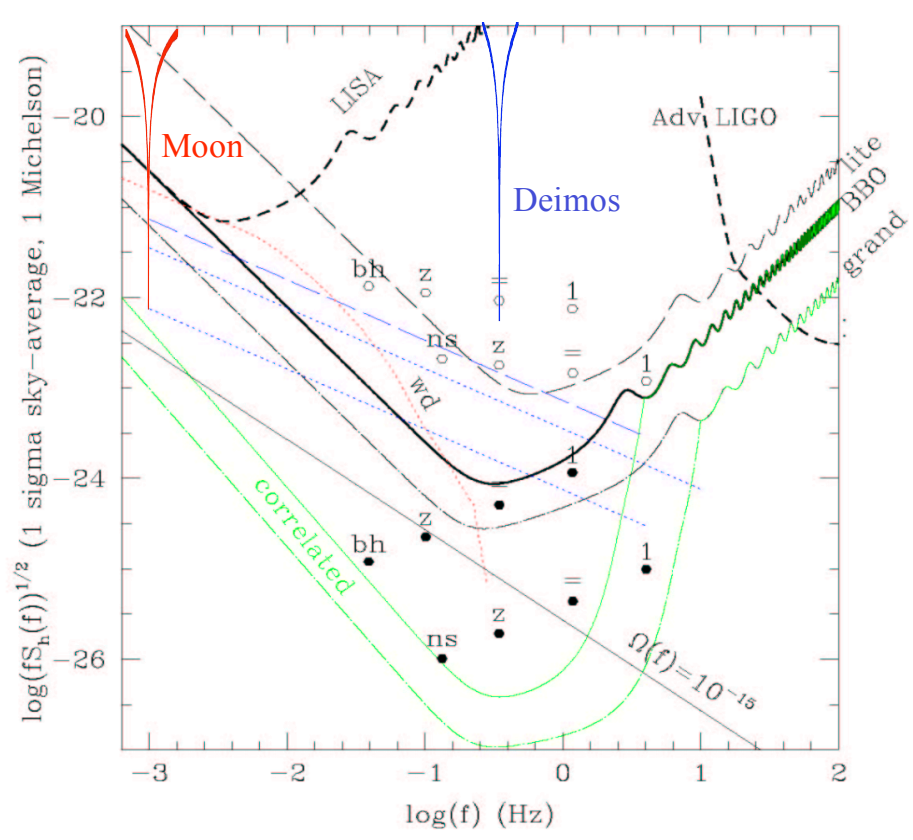

Figure 3. Sensitivities of the Moon and Deimos compared with LIGO, LISA and BBO. 


\section{Technology requirement}

A critical technology that needs to be developed to enable GW detection on the Moon or a Martian moon is a space-qualified "vibration-free" cryocooler for $T \leq 4 \mathrm{~K}$. Several types of closedcycle refrigerators are under development for applications in infrared astronomy missions. The pulse-tube cryocooler operates at $55 \mathrm{~K}$ and is almost free of vibration (Ross et al 1998). An additional cooling stage could be added to this cooler to reach $4 \mathrm{~K}$ and below. Another option of achieving vibration-free refrigeration is the reverse-Brayton cryocooler. The crycoolers are power-hungry. So it appears that RTGs (Radioisotope Thermal Generators) will be required to provide power continuously through the day-night cycle.

To obtain the projected sensitivity, the superconducting displacement sensor needs to be improved in two fronts. The high quality factor $\left(Q \approx 10^{8}\right)$ must be demonstrated in a magnetically levitated superconducting test mass. For low-frequency operation $(f<0.1 \mathrm{~Hz})$, it is desirable to upconvert the signal frequency to above the $1 / \mathrm{f}$ corner frequency of the SQUID. An ac bridge transducer is under development to achieve this goal (Chui et al 2004).

In addition to the cryocooler vibration, there are numerous other sources of seismic noise: moonquakes, thermal quakes at sunrise ( $>1 \mathrm{~Hz}$, diurnal), meteorite impacts, and human activities. The seismic noise is the most likely limiting error source, especially for experiments on the Moon. It is therefore important to develop a procedure to veto the seismic excitation by using the unique tensor nature of GW. It is highly desirable to survey the seismic background of the moons with superconducting displacement sensors prior to a GW experiment.

Gravity noise will arise from tidal deformation and thermal expansion of the ground and the apparatus. However, these are not of concern since they will occur at extremely low frequencies, well outside the signal bandwidth. The instrument could be buried in the regolith to reduce its temperature variation. The levitated test masses will be electrically charged by cosmic rays. This will also occur at very low frequencies outside the signal bandwidth.

\section{Acknowledgment}

We have benefited from discussions with Kip Thorne, Yanbei Chen, Sterl Phinney, Yosio Nakamura, Don Strayer, and Vol Moody. This work was supported by a NASA grant under NRA01-OBOR-08-E and by JPL through its appointment of one of us (HJP) as a Distinguished Visiting Scientist.

\section{References}

Chen Y and Thorne K S 2004 Private communication

Chui T et al $200435^{\text {th }}$ COSPAR Scientific Assembly, Paris, France

Goins N R, Dainty A M and Toksöz M N 1981 J. Geophys. Res. 86378

Johnson W W and Merkowitz S M 1993 Phys. Rev. Lett. 702367

Lammlein D R et al 1974 Rev. Geophys. Space Phys. 121

Lobo J A 1995 Phys. Rev. D 52591

Paik H J 1976 J. Appl. Phys. 471168

Phinney S 2004 Private communication

Ross R G 1998 Proceedings of the $10^{\text {th }}$ International Cryocooler Conference, Monterey, California

Wagoner R V and Paik H J 1977 Experimental Gravitation (Rome: Accademia Nazionale dei Lincei) p 257 